\title{
Metales pesados en humedales de arroz en la cuenca baja del río Guayas
}

\author{
Wilson Pozo ${ }^{1}$, Teófile Sanfeliu', Gloria Carrera ${ }^{3}$ \\ ${ }^{1}$ Dirección de Investigación y Proyectos Académicos. Universidad de Guayaquil. \\ Avenida Chile y Olmedo; edificio Posgrado; Guayaquil, Ecuador \\ ${ }^{2}$ Escuela Superior de Tecnología i Ciencias Experimentales. Universitat Jaume I, 12080, Castellón, \\ España \\ ${ }^{3}$ Instituto Nacional de Investigaciones Agropecuarias, Estación Experimental Litoral Sur, Ecuador \\ Autor para correspondencia:wpozo@espoltel.net
}

Fecha de recepción: 4 de junio de 2011 - Fecha de aceptación: 20 agosto de 2011

\section{RESUMEN}

La cuenca baja del río Guayas es una área de humedales costeros donde se cultiva arroz en una extensión de 350.000 ha. Se estableció un área experimental de $100 \mathrm{~km}^{2}$ a las dos orillas del río Babahoyo el mismo que es un tributario del río Guayas, correspondiente a los cantones Samborondón y Yaguachi. En 26 estaciones de muestreo se midió los metales pesados en suelos, su relación con las propiedades edáficas y su concentración en las plantas de arroz. Se recolectaron muestras de suelo en los 26 sitios, mientras que las plantas fueron monitoreadas en 5 estaciones de muestreo. La metodología empleada se baso en un análisis de las propiedades físicas, químicas de los suelos y sus relaciones mediante un análisis multivariado. Se determinó valores promedio de variables: MO 4\%, Arcilla 32,7\%, Limo 49,8\%, Arena 17,5\%, pH 6,6, CE 7,9 mS m${ }^{-1}$, metales en $\mathrm{mg} \mathrm{kg}^{-1} \mathrm{Cu} 48,8, \mathrm{Fe}$ 8.734, $\mathrm{Mn} 343, \mathrm{Zn} \mathrm{34,} \mathrm{Hg}$ no detectado, $\mathrm{Cd} 0,15$, y $\mathrm{Pb} 4,4$. Las variables evaluadas fueron 12 y se explicó el 40,7\% en los dos primeros componentes con los autovalores limo y arcilla (CP1) y arcilla y cadmio (CP2). Se observó que existe dispersión entre las localidades; se formaron seis grupos a una distancia euclidiana de 3,22 y algunos grupos presentan congruencia con el análisis de componentes principales. Se evidenció los niveles de metales pesados en suelos de acuerdo al siguiente orden: $\mathrm{Fe}>$ $\mathrm{Mn}>\mathrm{Cu}>\mathrm{Zn}>\mathrm{Pb}>\mathrm{Cd}$ y $\mathrm{Hg}$ no detectado. El plomo que se midio en cinco sitios presentó en valores promedio mínimos y máximos de $\mathrm{mg} \mathrm{kg}^{-1}$ : en suelos 6,55-8,87; el contenido de la planta de arroz: raíz 3,30-4,40; tallo 2,01-2,60; y hoja 1,80-2,00.

Palabras clave: Metales pesados, fitotoxicos, cultivo de arroz, cuencas.

\begin{abstract}
In the lower basin of Guayas, more in particular in the wetland area, the rice is cultivated on 350.000 ha. An area of $100 \mathrm{~km}^{2}$, covering both sides of the tributary river Babahoyo, situated in the cantons Samborondón and Yaguachi, was selected to study the presence of heavy metals in the top soil and plants. Soil samples were collected in 26 sites, whereas plants were sampled in 5 sampling stations. The concentration of heavy metals in the top soil was related to soil edaphological properties and the concentration in the rice plants, using multivariate analysis. At each site 12 variables were measured resulting over the 26 sites in the following average values: MO 4\%, clay 32,7\%, loam 49,8\%, sand 17,5\%, pH 6,6, CE 7,9 $\mathrm{mS} \mathrm{m}^{-1}$, metal concentration in $\mathrm{mg} \mathrm{kg}^{-1} \mathrm{Cu} 48,8, \mathrm{Fe} 8.734, \mathrm{Mn} \mathrm{343,} \mathrm{Zn} \mathrm{34,} \mathrm{Hg}$ not detected, $\mathrm{Cd} 0,15$ y $\mathrm{Pb} 4,4$. Statistical analysis revealed that $40,7 \%$ of the spatial variability in heavy metals in the top soil is explained by the first two principal components, loam and clay (CP1) and clay and cadmium (CP2). In addition, the 26 sites could be grouped in 6 distinct classes at an euclidian distance of 3,22. Based on the concentration the heavy metals in the top soil rank from high to low as follows: $\mathrm{Fe}>\mathrm{Mn}>\mathrm{Cu}>\mathrm{Zn}>\mathrm{Pb}>\mathrm{Cd}$ and $\mathrm{Hg}$ not detected. The concentration of lead in the sites where also the lead concentration was measured in the rice plants, 5 locations in total, ranged
\end{abstract}


in the top soil between $6,55-8,87 \mathrm{mg} \mathrm{kg}^{-1}$, in the roots between $3,30-4,40 \mathrm{mg} \mathrm{kg}^{-1}$, in the stems between 2,01-2,60 $\mathrm{mg} \mathrm{kg}^{-1}$, and in the leaves between 1,80 and $2,00 \mathrm{mg} \mathrm{kg}^{-1}$.

Keywords: Heavy metals, fytotoxicity, rice crop, basin.

\section{INTRODUCCIÓN}

El potencial agrícola del Ecuador está en el litoral ecuatoriano; sus principales cultivos son: arroz (350.000 ha), cacao (434.418 ha), café (320.911 ha), banano (216.124 ha), plátano (183.599 ha) y palma africana (162.212 ha). El arroz se lo cultiva en la cuenca media y baja en su mayor proporción (SICA, 2001). Los productos alimenticios obtenidos son destinados al mercado interno y de exportación los mismos que exigen que los productos sean libres de elementos nocivos, tales como metales pesados. Estos metales son absorbidos por los vegetales y de esta manera se encuentran disponibles para los herbívoros y humanos directamente o a través de la cadena alimentaria (John y Leventhal, 1995). En el 2009 se cultivaron en el Ecuador 373.624 ha, siendo la provincia del Guayas la de mayor extensión (203.649 ha), lo que constituye el 54,5\%, con una producción de 670.986 TM. La provincia de los Ríos se ubica en segundo lugar con una superficie sembrada de 125.595 ha, alcanzando el 33,1\% del total nacional y una producción de 379.438 TM de arroz seco limpio (Rodríguez, 2010).

El área de producción agrícola de la Cuenca del Guayas tiene problemas ambientales por la prescencia de metales pesados de naturaleza geogénica por la meteorización de la roca parental procedente de la Cordillera de los Andes y por actividad antropogénica por acumulación de residuos industriales, actividad minera, quema de basura y residuos agrícolas, explotación de petróleo y el uso indiscriminado de insumos químicos en la agricultura (Pozo, 2001; UEFC, 2002). Los suelos con cultivo de arroz de la Cuenca baja del río Guayas tienen un $\mathrm{pH}$ que varía desde muy fuertemente ácido $(4,5)$ a alcalino $(8,3)$, los mismos que presentan problemas por salinidad y contenido de materia orgánica, que está en el rango desde bajo $(1,8)$ a alto $(6,0)$. La textura varía desde arcilloso, francoarcilloso, limoso, franco-limoso hasta franco-arcillo-limoso (Pozo, 2010).

Según Gulson y col. (1996), las excesivas concentraciones de metales pesados en el suelo podrían impactar la calidad de los alimentos, la seguridad de la producción de cultivos y la salud debido a que éstos se mueven a través de la cadena alimenticia tanto vía consumo de plantas como por el consumo de animales que fueron alimentados con éstos productos. Los metales acumulados en los suelos se reducen lentamente mediante la lixiviación, el consumo por las plantas, la erosión y la deflación. Baird (1999) manifiesta que los metales pesados tienden a acumularse en la superficie del suelo quedando accesibles al consumo para las raíces de los cultivos. Las plantas cultivadas en suelos contaminados absorben mas oligoelementos y la concentración de estos en los tejidos vegetales está a menudo directamente relacionada con su abundancia en los suelos, especialmente en la solución húmeda que adsorbe la planta (Kabata-Pendias y Pendias, 2004).

El plomo $(\mathrm{Pb})$ es un metal pesado potencialmente tóxico, sin función biológica conocida, el mismo que ha atraído considerable atención por su amplia distribución y riesgo aleatorio para el medio ambiente. La contaminación por $\mathrm{Pb}$ en suelos cultivados con arroz no sólo despertó los cambios de microorganismos del suelo y sus actividades que dio como resultado el deterioro de la fertilidad del suelo, también afectó directamente el cambio de los índices fisiológicos del arroz, y más la decadencia del rendimiento y deterioro de la calidad del mismo (Majer y col., 2002). En última instancia, el plomo enriquecido en el cuerpo de los seres humanos a través de la cadena alimentaria, pone en peligro su salud (Liu y col., 2003). La actividad microbiana del suelo tiene un gran potencial como uno de los primeros indicadores sensible a las alteraciones en las propiedades de los suelos (Brookes, 1995), y ha sido empleado en programas de control a nivel nacional e internacional (Yao y col., 2000).

La contaminación por metales pesados del recurso tierra sigue siendo el objetivo de numerosos estudios ambientales y atrae la atención en todo el mundo. Esto se atribuye a la falta de biodegradabilidad y la persistencia de los metales pesados en los suelos (Adriano, 2001). Por lo tanto, 
la contaminación por metales pesados en suelos agrícolas y su transferencia en un sistema suelo-arroz han sido de preocupación creciente. Varios estudios sobre agricultura sostenible, informaron sobre la acumulación y la transferencia de metales pesados del suelo hacia los diferentes órganos de las plantas; así como la influencia de las propiedades del suelo y de la variación genotípica del arroz en la absorción de metales pesados. La distribución y acumulación de metales pesados en plantas se distribuye en sus órganos: raíz, tallo y hojas. Los granos presentaron contenidos significativamente más bajos que otros órganos (Kashem, 2001; Liu, 2003).

Los metales pesados se encuentran presentes en los suelos y en las plantas de arroz en la Cuenca baja del río Guayas. Este estudio presenta el contenido de metales pesados en suelos y su relación con las propiedades edáficas, y la concentración de los mismos en plantas de arroz. Los objetivos específicos del estudio fueron: (1) determinar por métodos analíticos convencionales la presencia y concentración de los metales pesados $(\mathrm{Cd}, \mathrm{Hg}, \mathrm{Pb}, \mathrm{Cu}, \mathrm{Fe}, \mathrm{Mg}, \mathrm{Zn})$ así como su relación con diversas propiedades edáficas, y (2) establecer la presencia de los metales fitotóxicos $(\mathrm{Cd}, \mathrm{Hg}, \mathrm{Pb})$ en la planta de arroz (Oryza sativa L.).

\section{MATERIALES Y MÉTODOS}

\subsection{Zona de estudio y muestreo}

Se tomaron muestras de suelos en 26 localidades o sitios de muestreo georeferenciados en la Cuenca del río Guayas en una extensión de $100 \mathrm{~km}$ a las dos orillas del río tributario Babahoyo, correspondiente a los cantones Samborondón y Yaguachi (Figura 1). Se colectaron las 26 muestras de suelos a una profundidad de 0 a $20 \mathrm{~cm}$. Las muestras fueron secadas al aire libre, trituradas en un mortero de agata y pasadas por un tamiz de $2 \mathrm{~mm}$.

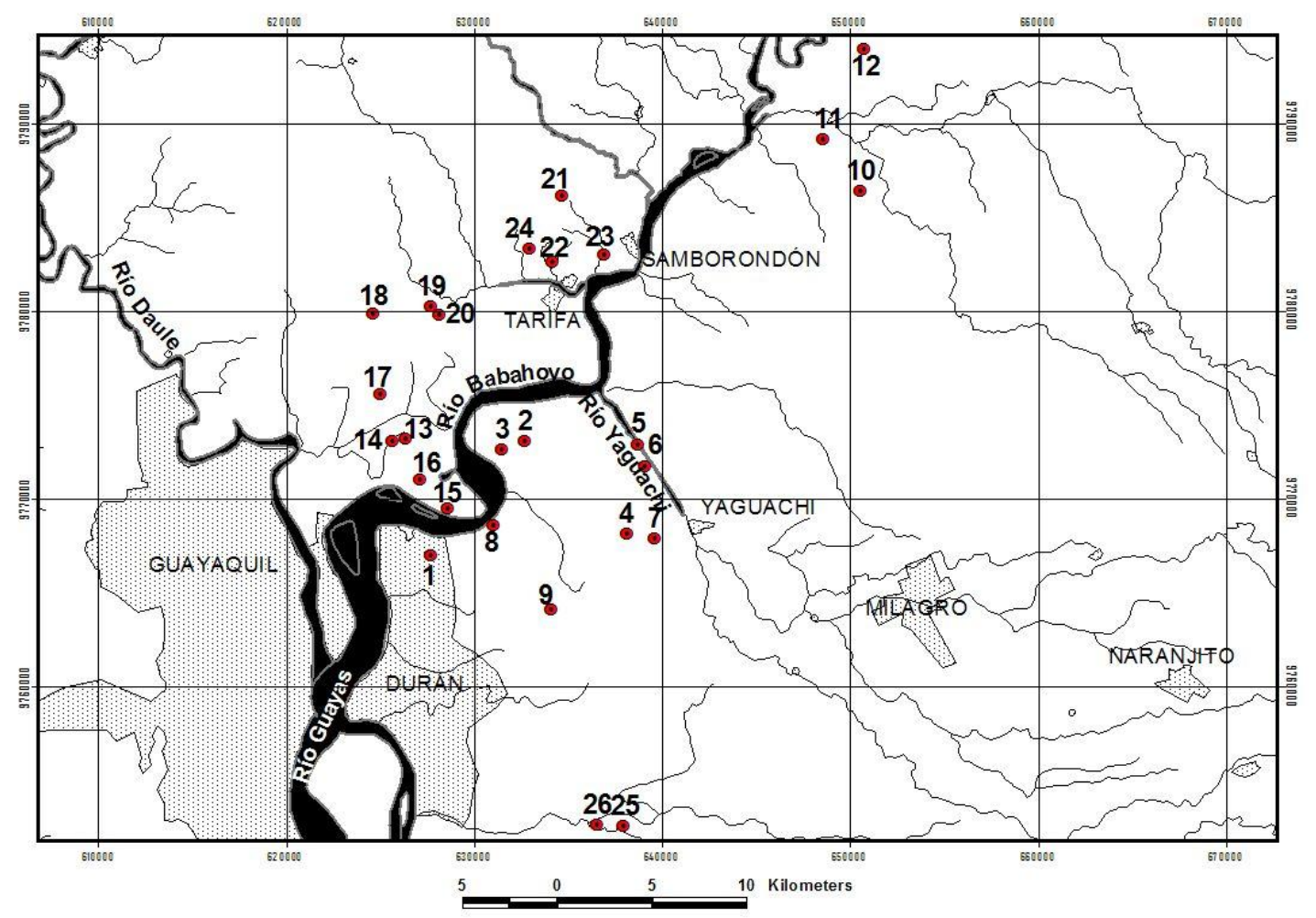

Figura 1. Ubicación de las estaciones de muestreo. 
MASKANA, Vol. 2, No. 1, 2011

Tabla 1. Propiedades físicas y químicas del suelo.

\begin{tabular}{|c|c|c|c|c|c|c|c|c|c|c|c|c|c|c|}
\hline \multirow{2}{*}{ \# } & \multirow{2}{*}{ Localidades de muestreo } & \multicolumn{4}{|c|}{ Contenido (\%) } & \multirow{2}{*}{$\mathrm{pH}$} & \multirow{2}{*}{$\frac{\mathrm{CE}}{\mathrm{mS} \mathrm{m^{-1 }}}$} & $\mathrm{Cu}$ & \multirow[t]{2}{*}{$\mathrm{Fe}$} & \multirow[t]{2}{*}{$\mathrm{Mn}$} & \multirow{2}{*}{$\frac{\mathrm{Zn}}{\mathrm{mg} \mathrm{kg}}$} & \multirow[t]{2}{*}{$\mathrm{Hg}$} & \multirow[t]{2}{*}{$\mathrm{Cd}$} & \multirow[t]{2}{*}{$\mathrm{Pb}$} \\
\hline & & MO & Arena & Limo & Arcilla & & & & & & & & & \\
\hline 1 & Coop. La Carmela & 1,9 & 8 & 88 & 4 & 8,2 & 3,7 & 35,1 & 5.310 & 250 & 24 & $<\mathrm{LC}$ & 0,11 & 3,8 \\
\hline 2 & María Clementina & 5,6 & 14 & 56 & 30 & 7,2 & 14,0 & 54,2 & 7.100 & 329 & 55 & $<\mathrm{LC}$ & 0,13 & 3,7 \\
\hline 3 & El Caliz & 5,0 & 17 & 47 & 36 & 6,3 & 14,5 & 68 & 7.360 & 481 & 27 & $<\mathrm{LC}$ & 0,16 & 6,6 \\
\hline 4 & Coop. 1001 & 4,9 & 20 & 74 & 6 & 7,0 & 8,9 & 44,3 & 6.320 & 256 & 30 & $<\mathrm{LC}$ & 0,08 & 8,1 \\
\hline 5 & Bodeguita & 4,0 & 22 & 52 & 26 & 7,2 & 5,9 & 52,1 & 6.501 & 274 & 33 & $<\mathrm{LC}$ & 0,12 & 8,3 \\
\hline 6 & San Alfonso & 4,0 & 44 & 26 & 30 & 6,5 & 5,3 & 73,3 & 8.760 & 341 & 26 & $<\mathrm{LC}$ & 0,20 & 3,1 \\
\hline 7 & Las Mercedes & 4,5 & 12 & 54 & 34 & 6,5 & 6,4 & 48,4 & 6.210 & 286 & 28 & $<\mathrm{LC}$ & 0,13 & 2,9 \\
\hline 8 & Guajala & 3,2 & 14 & 36 & 50 & 7,7 & 7,7 & 5,0 & 8.840 & 324 & 29 & $<\mathrm{LC}$ & 0,10 & 4,3 \\
\hline 9 & Casiguana & 2,8 & 18 & 28 & 54 & 7,1 & 17,6 & 33,6 & 7.050 & 281 & 31 & $<\mathrm{LC}$ & 0,10 & 3,6 \\
\hline 10 & La Ilusión & 3,4 & 18 & 68 & 14 & 7,4 & 2,1 & 60,0 & 13.832 & 300 & 21 & $<\mathrm{LC}$ & 0,39 & 1,9 \\
\hline 11 & Jesus María El Otoño & 6,1 & 32 & 64 & 4 & 7,7 & 1,3 & 50,6 & 11.128 & 452 & 56 & $<\mathrm{LC}$ & 0,20 & 2,1 \\
\hline 12 & El Porvenir- Aso. Sta. Elena & 3,5 & 14 & 44 & 42 & 6,7 & 1,2 & 38,2 & 9.646 & 540 & 26 & $<\mathrm{LC}$ & 0,04 & 3,0 \\
\hline 13 & $\begin{array}{l}\text { La Barranca Toma II Victor } \\
\text { Mora }\end{array}$ & 2,8 & 13 & 52 & 35 & 7,1 & 15,2 & 31,9 & 6.832 & 412 & 35 & $<\mathrm{LC}$ & 0,10 & 3,1 \\
\hline 14 & La Barranca Toma I C. Carranza & 6,1 & 24 & 35 & 41 & 5,5 & 11,9 & 51,2 & 11.960 & 420 & 42 & $<\mathrm{LC}$ & 0,16 & 3,2 \\
\hline 15 & Sabanilla & 4,2 & 12 & 42 & 46 & 5,2 & 5,0 & 47,4 & 8.892 & 486 & 28 & $<\mathrm{LC}$ & 0,15 & 3,1 \\
\hline 16 & San Nicolas & 5,0 & 17 & 76 & 7 & 6,4 & 25,7 & 53,8 & 9.048 & 408 & 33 & $<\mathrm{LC}$ & 0,21 & 8,9 \\
\hline 17 & Coop. Buijo & 3,7 & 8 & 32 & 60 & 6,1 & 20,2 & 40,6 & 7.270 & 308 & 38 & $<\mathrm{LC}$ & 0,16 & 3,4 \\
\hline 18 & Coop. General Gómez E. Lorenti & 2,8 & 24 & 42 & 34 & 7,9 & 3,7 & 38,2 & 16.354 & 298 & 49 & $<$ L.C. & 0,13 & 3,3 \\
\hline 19 & Coop. General Gómez C. Barzola & 4,8 & 12 & 34 & 54 & 5,7 & 3,1 & 35,1 & 15.834 & 384 & 37 & $<\mathrm{LC}$ & 0,19 & 8,6 \\
\hline
\end{tabular}


MASKANA, Vol. 2, No. 1, 2011

Tabla 1: Propiedades físicas y químicas del suelo (continuación).

\begin{tabular}{|c|c|c|c|c|c|c|c|c|c|c|c|c|c|c|}
\hline \multirow{2}{*}{$\#$} & \multirow{2}{*}{ Localidades de muestreo } & \multicolumn{4}{|c|}{ Contenido (\%) } & \multirow{2}{*}{$\mathrm{pH}$} & \multirow{2}{*}{ 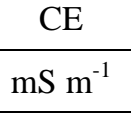 } & $\mathrm{Cu}$ & \multirow[t]{2}{*}{$\mathrm{Fe}$} & \multirow[t]{2}{*}{$\mathrm{Mn}$} & \multirow{2}{*}{$\frac{\mathrm{Zn}}{\mathrm{mg} \mathrm{kg}^{-1}}$} & \multirow[t]{2}{*}{$\mathrm{Hg}$} & \multirow[t]{2}{*}{$\mathrm{Cd}$} & \multirow[t]{2}{*}{$\mathrm{Pb}$} \\
\hline & & MO & Arena & Limo & Arcilla & & & & & & & & & \\
\hline 20 & Coop. General Gómez E. Diaz & 3,1 & 20 & 36 & 44 & 5,8 & 3,1 & 56,7 & 9.150 & 268 & 41 & $<\mathrm{LC}$ & 0,18 & 4,4 \\
\hline 21 & Gran Colombia -Cerro & 5,0 & 8 & 36 & 56 & 5,5 & 6,3 & 75,2 & 10.166 & 294 & 44 & $<\mathrm{LC}$ & 0,19 & 5,3 \\
\hline 22 & Lupercio Franco & 3,2 & 10 & 38 & 52 & 6,5 & 3,2 & 39,7 & 6.643 & 472 & 30 & $<\mathrm{LC}$ & 0,15 & 2,8 \\
\hline 23 & Flor María & 6,3 & 22 & 40 & 38 & 6,5 & 1,8 & 40,6 & 6.230 & 298 & 32 & $<\mathrm{LC}$ & 0,08 & 3,5 \\
\hline 24 & Germania Yulan & 1,9 & 10 & 86 & 4 & 7,3 & 6,0 & 37,6 & 4.100 & 196 & 24 & $<\mathrm{LC}$ & 0,14 & 4,5 \\
\hline 25 & Vencedores de Yamile & 3,9 & 16 & 78 & 6 & 5,8 & 6,4 & 47,2 & 6.042 & 234 & 26 & $<\mathrm{LC}$ & 0,12 & 3,5 \\
\hline \multirow[t]{5}{*}{26} & Coop. Ataulfo Cruz & 2,6 & 26 & 32 & 42 & 5,0 & 4,1 & 59,0 & 10.504 & 326 & 36 & $<\mathrm{LC}$ & 0,10 & 4,2 \\
\hline & Promedio & 4,0 & 17,5 & 49,8 & 32,7 & 6,6 & 7,9 & 48,8 & 8.734 & 343 & 34 & ND & 0,15 & 4,4 \\
\hline & Minima & 1,9 & 8,0 & 32,0 & 4,0 & 5,0 & 1,2 & 31,9 & 4.100 & 196 & 21 & ND & 0,04 & 1,9 \\
\hline & Maxima & 6,3 & 32,0 & 88,0 & 56,0 & 8,2 & 25,7 & 75,2 & 16.354 & 540 & 56 & ND & 0,39 & 8,9 \\
\hline & $\mathrm{CV} \%$ & 31,2 & 46,5 & 37,1 & 56,2 & 13,6 & 81,3 & 24,5 & 35,5 & 26,1 & 27,3 & ND & 0,44 & 46,0 \\
\hline
\end{tabular}

Leyenda: $\mathrm{MO}=$ la materia orgánica; $\mathrm{pH}=$ acidez $\mathrm{CE}=$ conductividad eléctrica $\mathrm{LC}=$ limite de cuantificación; $\mathrm{ND}=$ no detectado; $\mathrm{CV}=$ coeficiente de variación 


\subsection{Propiedades físicas y químicas del suelo, y los contenidos de metales en arroz}

Las propiedades físicas, químicas y los contenidos de metales en las plantas de arroz, se presentan en la Tabla 1 y 2. La Tabla 1 muestra los valores medidos de los 12 parametros tanto físicos como químicos (pH, MO, \% de arcilla, limo y arena, $\mathrm{CE}$, y metales $\mathrm{Cu}, \mathrm{Fe}, \mathrm{Mn}, \mathrm{Zn}, \mathrm{Hg}, \mathrm{Cd}$ y Pb) para los suelos entre 0 y $20 \mathrm{~cm}$ de profundidad. La concentration de $\mathrm{Hg}$ en todas las muestras analizadas es fue menor al limite de detección mientras los valores de Cd fueron bajos en rango de un 0,04 a 0,44 mg $\mathrm{kg}^{-1}$, la concentracion de $\mathrm{Pb}$ vario entre 1,90 e $8,87 \mathrm{mg} \mathrm{kg}^{-1}$. En las 26 muestras se obtuvo un $\mathrm{pH}$ promedio de 6,6 con un mínimo de 5,0 y un máximo de 8,2, con un coeficiente de variación de 13,6. La materia orgánica (MO) con promedio de $4 \%$ y un valor mínimo de $1,9 \%$ y un máximo de $6,3 \%$, y con un coeficiente de variación de $31,2 \%$. La textura de los suelos esta conformada por (i) arena con un valor promedio de $17,5 \%$, un valor mínimo de $8,0 \%$, un valor máximo de $32,0 \%$, y un CV de 46,5\%; (ii) el limo con un promedio de 49,8\%, un valor mínimo de 31,0 , un valor máximo de $88,0 \%$ y un CV de 37,1\%; y (iii) arcilla con un valor promedio de 32,7\%, un valor mínimo de 4,0\%, un valor máximo de 56,0\%, y un CV de 56.2\%. Mientras los valores de Cd son bajos en un rango de 0,04 a $0,44 \mathrm{mg} \mathrm{kg}^{-1}$, la concentracion de $\mathrm{Pb}$ varia entre 1,90 y $8,87 \mathrm{mg} \mathrm{kg}^{-1}$.

Tabla 2. Descripción estadística de los contenidos de plomo en suelos y arroz en cinco localidades.

\begin{tabular}{|c|c|c|c|c|c|c|}
\hline \multirow{2}{*}{$\#$} & \multirow{2}{*}{ Localidades de muestreo } & \multirow{2}{*}{$\begin{array}{l}\text { Parámetro } \\
\text { estadístico }\end{array}$} & Suelo & Raíz & Tallo & Hoja \\
\hline & & & \multicolumn{4}{|c|}{$\mathrm{Pb}\left(\mathrm{mg} \mathrm{kg}^{-1}\right)$} \\
\hline \multirow[t]{4}{*}{3} & \multirow[t]{4}{*}{ Los Cáliz } & Promedio & 6,55 & 3,76 & 2,01 & 1,94 \\
\hline & & Mínimo & 6,14 & 3,26 & 1,82 & 1,82 \\
\hline & & Máximo & 7,12 & 4,24 & 2,26 & 2,10 \\
\hline & & $\mathrm{CV}[\%]$ & 7,70 & 13,00 & 11,00 & 7,40 \\
\hline \multirow[t]{4}{*}{4} & \multirow[t]{4}{*}{ Cooperativa 1001} & Promedio & 8,01 & 4,58 & 2,69 & 1,82 \\
\hline & & Mínimo & 7,54 & 4,11 & 2,50 & 1,62 \\
\hline & & Máximo & 8,50 & 5,15 & 2,97 & 2,10 \\
\hline & & $\mathrm{CV}[\%]$ & 6,00 & 12,00 & 9,00 & 13,70 \\
\hline \multirow[t]{4}{*}{5} & \multirow[t]{4}{*}{ Bodeguita } & Promedio & 8,29 & 4,47 & 2,31 & 1,80 \\
\hline & & Mínimo & 7,70 & 3,90 & 2,13 & 1,60 \\
\hline & & Máximo & 8,76 & 5,10 & 2,50 & 2,00 \\
\hline & & $\mathrm{CV}[\%]$ & 6,50 & 13,00 & 8,00 & 11,10 \\
\hline \multirow[t]{4}{*}{16} & \multirow[t]{4}{*}{ San Nicolas } & Promedio & 8,87 & 4,67 & 2,51 & 1,80 \\
\hline & & Mínimo & 8,50 & 4,20 & 2,32 & 1,63 \\
\hline & & Máximo & 9,40 & 5,14 & 2,80 & 1,93 \\
\hline & & $\mathrm{CV}[\%]$ & 5,30 & 10,00 & 10,00 & 8,50 \\
\hline \multirow[t]{4}{*}{19} & \multirow[t]{4}{*}{ General Gómez } & Promedio & 8,59 & 3,30 & 2,60 & 2,00 \\
\hline & & Mínimo & 8,07 & 3,10 & 2,40 & 1,80 \\
\hline & & Máximo & 9,10 & 3,60 & 2,80 & 2,30 \\
\hline & & $\mathrm{CV}[\%]$ & 6,00 & 8,00 & 8,00 & 13,20 \\
\hline
\end{tabular}

En la Tabla 2 se presenta la descripción estadística de los contenidos de $\mathrm{Pb}$ en suelos y arroz en cinco localidades evaluadas, donde se recolectaron y analizaron muestras de suelos, raíz, tallo y hojas con la finalidad de investigar como el $\mathrm{Pb}$, se presenta en el suelo, pasa al tallo y las hojas mediante la absorción de la planta a través de las raíces. Los valores promedios en los suelos están entre 5,30 y $8,59 \mathrm{mg} \mathrm{kg}^{-1}$; en las raíces se encuentra entre 3,26 y $13,0 \mathrm{mg} \mathrm{kg}^{-1}$; en el tallo entre 1,82 y $11,0 \mathrm{mg}$ $\mathrm{kg}^{-1}$; y finalmente en las hojas entre 1,82 y $13,20 \mathrm{mg} \mathrm{kg}^{-1}$ mediante estos valores se estableció la presencia de plomo en plantas de arroz. 


\subsection{Métodos}

En las muestras recolectadas y preparadas se realizaron las siguientes determinaciones: $\mathrm{pH}$ según Henríquez y Cabalceta (1999), materia orgánica oxidable se estableció mediante el método rápido de Walkley y Black (1934), la textura se realizó por el método areómetro de Bouyouco (Jackson, 1970), conductividad eléctrica por el método del extracto de saturación (Allison, 1973; Bonnet, 1960), la concentración de Hierro (Fe), Manganeso (Mn), Zinc ( $\mathrm{Zn})$; Cobre (Cu); Plomo (Pb); Cadmio (Cd) y Mercurio (Hg). La técnica de espectrometría de absorción atómica (Perklin Elmer Analyst 700) fue utilizada para determinar la concentración de los elementos metálicos en la muestras. El análisis de $\mathrm{Pb}$ en plantas se realizó usando la técnica de digestión cerrada (microondas Marx-5) con ácido nítrico (AOAC, 2005; EPA, 1994). Para garantizar la calidad en los resultados analíticos, las muestras fueron analizadas por duplicado.

$\mathrm{El}$ análisis de $\mathrm{Cd}$ y $\mathrm{Pb}$ se realizó mediante espectrofotometría de absorción atómica con horno de grafito, en un equipo de Perkin Elmer Analyst 700, provisto de un automuestreador. El Hg se analizó por método de espectrometría de absorción atómica por vapor frío (CVAAS), y el Fe, $\mathrm{Cu}, \mathrm{Mn}$ y Zn se determinaron por absorción atómica. El límite de detección y límite de cuantificación de los elementos en estudio son: $\mathrm{Cu} 0,01 \mathrm{mg} \mathrm{l}^{-1}, 0,1 \mathrm{mg} \mathrm{kg}^{-1} ; \mathrm{Fe} 0,02 \mathrm{mg} \mathrm{l}^{-1}, 0,30 \mathrm{mg} \mathrm{kg}^{-1} ; \mathrm{Pb} 0,00009 \mathrm{mg} \mathrm{l}^{-1}$,

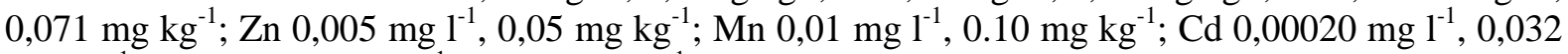
$\mathrm{mg} \mathrm{kg}{ }^{-1} ; \mathrm{Hg} 0,0001 \mathrm{mg} \mathrm{l}^{-1}, 0,09 \mathrm{mg} \mathrm{kg}^{-1}$; respectivamente. Analítico de digestión ácida fueron evaluados mediante el patrón de referencia RTC Catalogo \# CRM 008-050, Lote \# J408.

Los análisis estadísticos que se realizaron fueron: (1) análisis de componentes principales (ACP); (2) el método de la distancia euclidiana y el método de agrupamiento de ligamiento promedio para agrupar localidades; y (3) en función de los parámetros de caracterización de los suelos se realizaron los análisis solo con las variables que tienen relación con elementos químicos y/o metales pesados como $\mathrm{Cd}, \mathrm{Pb}, \mathrm{Cu}, \mathrm{Fe}, \mathrm{Mg}$ y $\mathrm{Zn}$. También se hicieron análisis de variancia en un diseño de bloques completos al azar para el contenido de plomo en el suelo y raíces, tallos y hojas de plantas de arroz muestreadas en cinco localidades: 3, 4, 5, 16 y 19 de la zona de estudio.

\section{RESULTADOS Y DISCUSIÓN}

\subsection{Concentraciones de metales pesados en los suelos}

Los resultados obtenidos en las determinaciones de los análisis en suelos de los metales traza en el perfil de 0 a $20 \mathrm{~cm}$ de profundidad para las 26 localidades de muestreo se puede apreciar en la Figura 2.

Los valores son los siguientes:

- La concentración de Cu tiene un promedio $48,8 \mathrm{mg} \mathrm{kg}^{-1}$ el punto mínimo es de $31,9 \mathrm{mg} \mathrm{kg}^{-1}$ el punto máximo es de 75,2 $\mathrm{mg} \mathrm{kg}^{-1}$ el coeficiente de variación es de 24,5\%;

- $\quad$ El Fe que tiene un promedio de $8.734 \mathrm{mg} \mathrm{kg}^{-1}$ el valor mínimo es de $4.100 \mathrm{mg} \mathrm{kg}^{-1}$, y un máximo de $16.354 \mathrm{mg} \mathrm{kg}^{-1}$ de concentración con un coeficiente de variación de 35,5\%;

- El Mn con promedio de $343 \mathrm{mg} \mathrm{kg}^{-1}$ de concentración con un mínimo de $196 \mathrm{mg} \mathrm{kg}^{-1}$ y un máximo de $540 \mathrm{mg} \mathrm{kg}^{-1}$ y un coeficiente de variación de $26,1 \%$;

- La concentración del $\mathrm{Zn}$ en el suelo con un promedio de $33,9 \mathrm{mg} \mathrm{kg}^{-1}$ los puntos mínimo y máximo 21 y $56 \mathrm{mg} \mathrm{kg}^{-1}$ respectivamente, con coeficiente de variación $27,3 \%$; y

- En $\mathrm{Hg}$ en el análisis no se detecto (ND) y el Cd con un promedio $0,15 \mathrm{mg} \mathrm{kg}^{-1}$, el mínimo con 0,04 y máximo con $0,39 \mathrm{mg} \mathrm{kg}^{-1}$ con un coeficiente de variación de 44,0\%; y el plomo con un promedio de $4,35 \mathrm{mg} \mathrm{kg}^{-1}$ y el mínimo de $1,90 \mathrm{mg} \mathrm{kg}^{-1}$ y el máximo de $8,87 \mathrm{mg} \mathrm{kg}^{-1}$, y un coeficiente de variación de $46,0 \%$. 

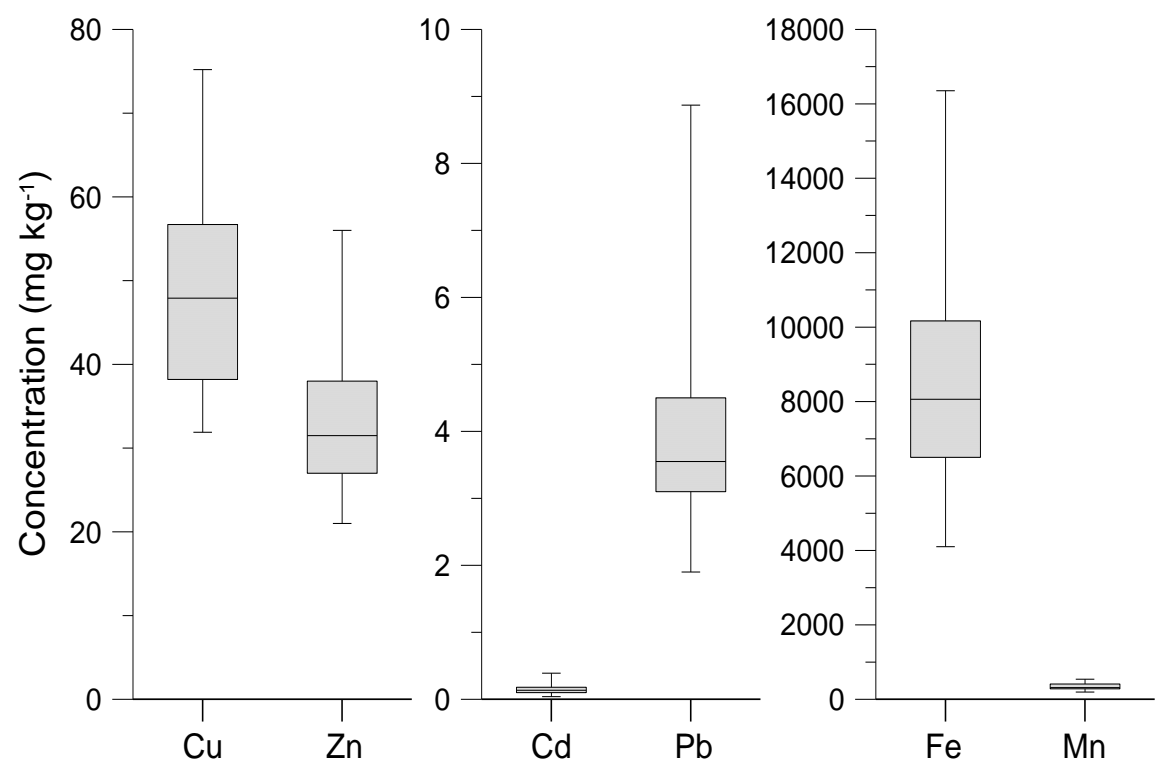

Figura 2. Diagrama de caja de bigotes de la concentración de $\mathrm{Cu}, \mathrm{Zn}, \mathrm{Cd}, \mathrm{Pb}, \mathrm{Fe}$ and $\mathrm{Mn}$ de 26 localidades de la zona de estudio.

Los metales pesados se definen como los metales de la tabla periódica con número atómico mayor a 20, excluyendo metales álcalis y tierras álcalis o metales que tienen densidades especificas mayores a $5 \mathrm{~g} \mathrm{~cm}^{-3}$ (Brunetti $y$ col., 2009). Las concentraciones de estos metales pueden incrementarse debido a variaciones geoquímicas locales o actividad antropogénicas de modo que se vuelven tóxicos para los organismos vivos incluyendo el ser humano (Ross, 1994). La quema de combustibles fósiles, incrementa el contenido de $\mathrm{As}, \mathrm{Cd}, \mathrm{Cu}, \mathrm{Ni}, \mathrm{Pb}, \mathrm{Se}, \mathrm{V}$ y $\mathrm{Zn}$ en suelos (Cataldo y col., 1987). La movilidad y la biodisponibilidad de los metales están determinadas por factores ambientales, y la fijación de metales en los sedimentos ocurre por adsorción y reacciones con la materia orgánica, debido al tipo de suelos, la textura, capacidad de intercambio catiónico y de retención, no obstante las arcillas exhiben alta retención por absorción (Brunetti y col., 2009). Los metales pesados pueden participar en una serie de procesos, incorporándose al ciclo del agua, principalmente en la fase relacionada con el suelo y el agua subterránea, puede acumularse en el suelos resultado de reacciones químicas vía procesos de adsorción, solubilización, precipitación y cambio en su estado de oxidación, o bien pueden estar presentes en tejidos vegetales, debido a su asimilación por las plantas (Navarro y col., 2007). Las consecuencias ecológicas en los suelos contaminados por metales pesados, aparentemente pasan desapercibidos, pero son potencialmente peligrosos para la vida terrestre (Bernal y col., 2007).

El Cd no tiene función biológica esencial y es altamente tóxico par plantas y animales, sin embargo, las cantidades de cadmio en el ambiente no causan toxicidad grave. El cadmio se absorbe más fácilmente sobre la superficie del suelo que otros metales. Se ha encontrado que este ion predomina el $\mathrm{pH}$ arriba de 6,5 como sulfato de cadmio o cloruro de cadmio. El equilibrio dinámico entre cadmio y suelo depende del $\mathrm{pH}$, la naturaleza química del metal, la estabilidad de complejos, fuerza de enlace de los grupos funcionales y la competencia con otros iones metálicos (Estévez y col., 2000). El plomo es un metal potencialmente dañino que tiende a contaminar el ambiente ya que se acumula en los suelos a causa de su baja solubilidad y relativa inmunidad a la degradación microbiológica por lo que permanece accesible a la cadena alimentaria y hay evidencias de que juegue un rol esencial en el metabolismos humano (Estévez y col., 2000). El Zn es un elemento traza esencial para las plantas superiores y los animales (Montenegro y col., 2009). Está presente en muchos alimentos y en suelos con bajo contenido de materia orgánica. Cobre $(\mathrm{Cu})$ es considerado uno de los elementos más importantes y esenciales para las plantas. Está asociado con la materia orgánica del suelo. La disponibilidad de éste elemento en las plantas se refiere a la facilidad con la que se es absorbido por las plantas (Torres y col., 2007). 


\subsection{Análisis multivariado con todas las variables evaluadas -elementos químicos y/o metales pesados}

Al realizar los análisis de componentes principales y de conglomerados solo con las variables que tienen relación con elementos químicos y/o metales pesados como $\mathrm{Cd}, \mathrm{Pb}, \mathrm{Cu}, \mathrm{Fe}, \mathrm{Mg}$ y $\mathrm{Zn}$. de acuerdo a los autovalores (Tabla 3), la mayor variación en CP1 la explican el hierro y el cadmio, este elemento también fue importante en el análisis con todas las variables; en el CP2 la variación la explican mayormente el cobre y manganeso (en sentido negativo).

En la Figura 3 se representan las localidades en las dos primeras componentes y se observa que la localidad 10 presenta el mayor contenido de cadmio mientras que las localidades 9,12 y 13 muestran los menores contenidos, similar a lo ya indicado con base al análisis de todas las variables (Figura 2). También se puede observar que las localidades 11 y 18 se caracterizan por sus valores altos de hierro y zinc. En el análisis de conglomerados se conformaron cinco grupos a una distancia de 2,75, únicamente las localidades 10, 18 y 19 no se agruparon. Los grupos en general tienen congruencia con la dispersión observada en el análisis de componentes principales.

Tabla 3. Autovalores de las dos primeras componentes principales del análisis con todas las variables evaluadas.

\begin{tabular}{ccc}
\hline \multirow{2}{*}{$\begin{array}{c}\text { Variables } \\
\text { originales }\end{array}$} & \multicolumn{2}{c}{ Componentes principales } \\
\cline { 2 - 3 } & $\mathrm{CP} 1$ & $\mathrm{CP} 2$ \\
\hline $\mathrm{Cu}$ & 0.41 & 0.50 \\
$\mathrm{Fe}$ & 0.60 & -0.22 \\
$\mathrm{Mn}$ & 0.25 & -0.49 \\
$\mathrm{Zn}$ & 0.34 & -0.44 \\
$\mathrm{Cd}$ & 0.54 & 0.39 \\
$\mathrm{~Pb}$ & -0.05 & 0.35 \\
\hline
\end{tabular}

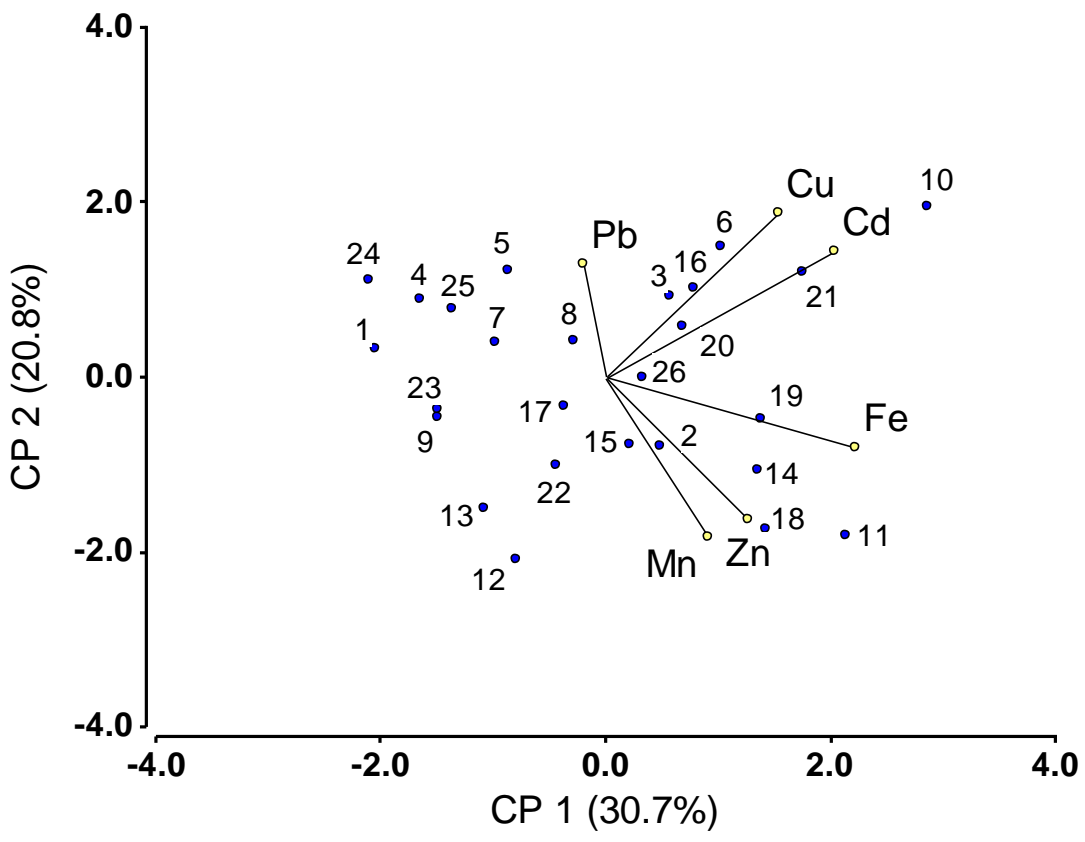

Figura 3. Representación de las dos primeras componentes principales y de las localidades en evaluación. Análisis con elementos químicos y/o metales pesados.

Los grupos o familias se forman en relacion con similitudes en la textura que son franco y arcillo en el primer grupo de las localidades $10,19,18,14,11$ y 2 . El pH esta en un rango de 5,5 a 7,9, la 
MO esta en un rango de 2,8 a 6,1\%, la conductividad electrica está de 1,3 a 13,95 $\mathrm{mS} \mathrm{m}^{-1}$, el Cd en un rango de 0,13 a $0,39 \mathrm{mg} \mathrm{kg}^{-1}$, y el $\mathrm{Pb}$ de 1,9 a $8,6 \mathrm{mS} \mathrm{m}^{-1}$. En el grupo conformado por las localidades $21,6,16$ y 3 presenta una textura franco a arcilloso, el pH está entre 5,5 a 7,2, la MO de 4 a 5\%, la conductividad eléctrica de 5,3 a $25,7 \mathrm{mS} \mathrm{m}^{-1}$, el Cd de 0,16 a $0,21 \mathrm{mg} \mathrm{kg}^{-1}$, y el Pb de 3,1 a $8,87 \mathrm{mg}$ $\mathrm{kg}^{-1}$. En el grupo que tiene la mayoría de localidades $(22,15,13,12,5,4,20,17,26,8,23,9,25,7$, 24 y 1) el pH es de 5,0 a 8,2, la conductividad eléctrica de 1,2 a $20,2 \mathrm{mS} \mathrm{m}^{-1}$, el Cd de 0,04 a $18 \mathrm{mg}$ $\mathrm{kg}^{-1}$, y el Pb tiene valores de 2,8 a $8,3 \mathrm{mg} \mathrm{kg}^{-1}$.

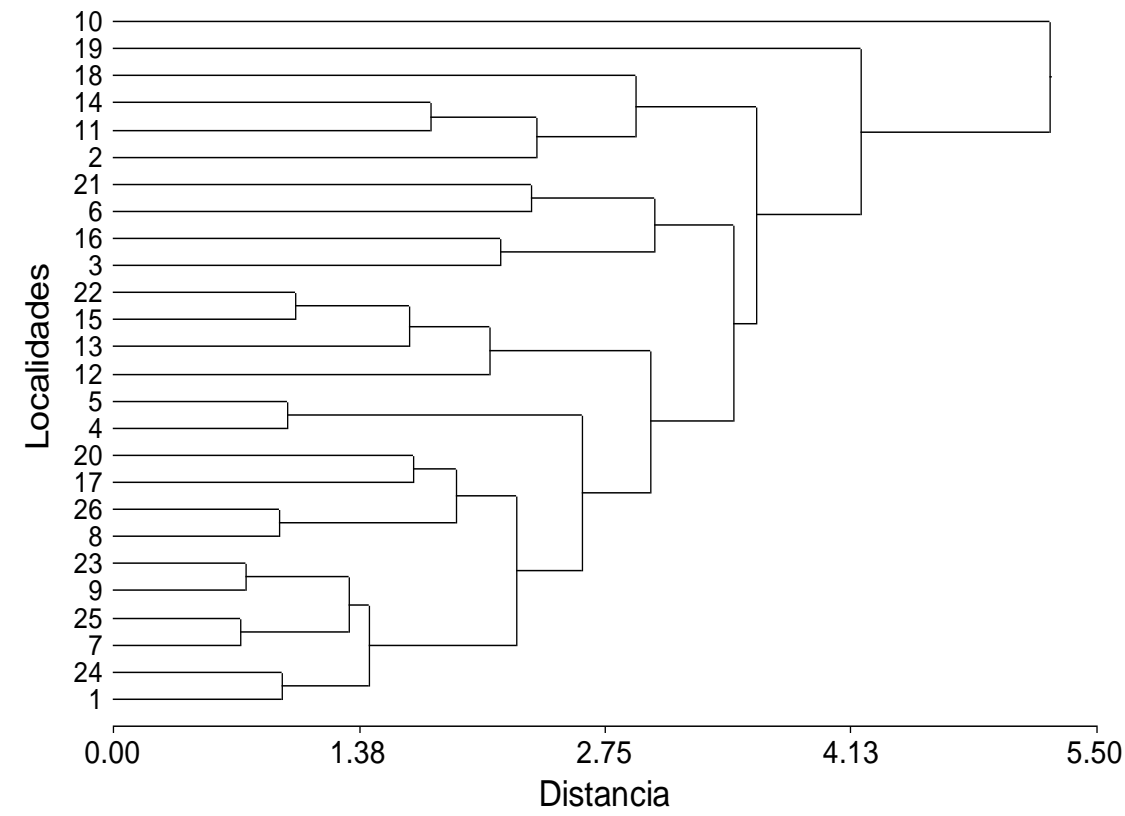

Figura 4. Análisis de conglomerados de las localidades en funcion de los elementos químicos y/o metales pesados.

\subsection{Anális de variancia para contenidos de Pb en suelo, raíz, tallo y hoja de arroz evaluadas en cinco localidades}

Se presentan los cuadrados medios de los análisis de variancia para contenido de plomo en el suelo y raíces, tallos y hojas de plantas de arroz, muestreadas en cinco localidades de la zona de estudio, observándose que, en cuanto al contenido en el suelo existen diferencias altamente significativas entre localidades. Al analizar el contenido en las plantas de arroz, se determinaron diferencias significativas entre localidades, al estudiar las raíces y tallos; mientras que, no se observaron diferencias entre localidades al analizar el contenido de plomo en las hojas.

Los promedios para los contenidos de plomo en suelo de las cinco localidades, así como en raíces, tallos y hojas de plantas de arroz tomadas en esas localidades se muestran en la Tabla 4. En cuanto al contenido en suelo, la localidad 3 presentó el promedio más bajo con $6,55 \mathrm{mg} \mathrm{kg}^{-1}$ y corresponde a la textura franco-arcillo-limoso; las localidades 4 y 5 a franco-limoso y la localidad 19 presenta una textura arcillosa, con valores correspondientes de $8,01,8,29$ y $8.59 \mathrm{mg} \mathrm{kg}^{-1}$ de plomo, mientras que la localidad 16 presentó el mayor contenido con $8,87 \mathrm{mg} \mathrm{kg}^{-1}$ y corresponde a la textura franco-limoso. La localidad 16 recibe la influencia directa de la marea que ingresa por el Río Babahoyo, de donde captan el agua para el riego del cultivo de arroz. Estos resultados indican que la concentración está en función de la distancia de las tomas de agua, la cual tiene la influencia de los vertidos de sustancias con plomo de la ciudad de Guayaquil. Otra relación que se aprecia es la textura de los suelos en función al tamaño de las partículas, presentando valores altos en el caso de los suelos de textura franco-limoso y arcilloso. Esta relación está en concordancia con lo manifestado por Pérez y col. (1995). Que señala que la textura, tiene una función fundamental en la dinámica de los metales pesados en los suelos. 
También hay una posible influencia de las cenizas volcánicas producidas por las erupciones del volcán Tungurahua que las viene emanando desde 1989, debido a que el área de estudio está en el curso de la caída de cenizas. En muestras colectadas en el área de estudio en análisis efectuado en cenizas volcánicas que caen en el área por la erupción del Tunguragua, estas cenizas tienen $2 \mathrm{mg} \mathrm{kg}^{-1}$. De acuerdo al Servicio de Estudios de la Contaminación de Cenizas y Suelos en la Ciudad de Chaltén y las propuestas de medidas de mitigación y restauración, estudio que reportan en Análisis de Cenizas Volcánicas valores mínimo 2 y máximo de $35 \mathrm{mg} \mathrm{kg}^{-1}$. Por otra parte, los bajos contenidos de fósforo en los suelos con valores de 5 a $9 \mathrm{mg} \mathrm{kg}^{-1}$, hace que se realice la fertilización con superfosfato de fósforo que en su composición contiene $7,2 \mathrm{mg} \mathrm{kg}^{-1} \mathrm{de} \mathrm{Pb}$. Para el caso del nitrógeno los contenidos varían de 15 a $29 \mathrm{mg} \mathrm{kg}^{-1}$ en los suelos estudiados (Pozo y col., 2010), siendo deficitarios en este elemento; para la fertilización se utiliza la urea como fuente de nitrógeno y esta contiene $1 \mathrm{mg} \mathrm{kg}^{-1} \mathrm{~Pb}$ según Wilke y Döhler (1995). Como la actividad de fertilización se la realiza de forma continua y al ser el $\mathrm{Pb}$ un metal que se fija al suelo y su elevada estabilidad, toxicidad, su acción es acumulativa en las cadenas alimenticias por lo que pueden llegar concentraciones muy altas en algunos órganos de los últimos constituyentes de las cadenas tróficas (red trófica), que muchas veces sirve para alimento para el hombre (Hernández y col., 1990). En estudio realizado por Arcos y Castro (2005) en la cuenca del río Taura, en sedimentos de 11 estaciones de muestreo la distribución de $\mathrm{Pb}$ no mostró un patrón definido, sus concentraciones varían de 9 a $33 \mathrm{mg} \mathrm{kg}^{-1}$ y en aguas los contenidos son de 0,05 a 0,38 $\mathrm{mg} \mathrm{l}^{-1}$.

Tabla 4. Promedios para contenido de plomo $\left(\mathrm{mg} \mathrm{kg}^{-1}\right)$ en suelos y raíces, tallos y hojas de plantas de arroz evaluadas en cinco localidades.

\begin{tabular}{cllllll}
\hline Localidad & Suelo & Raíz & \multicolumn{2}{c}{ Tallo } & Hojas \\
\hline 3 & $6,55 \mathrm{a}^{\dagger}$ & $3,76 \mathrm{ab}$ & $2,01 \mathrm{a}$ & 1,94 & $\mathrm{a}$ \\
4 & $8,01 \mathrm{~b}$ & $4,58 \mathrm{~b}$ & $2,69 \mathrm{c}$ & 1,82 & $\mathrm{a}$ \\
5 & $8,29 \mathrm{bc}$ & $4,47 \mathrm{~b}$ & $2,31 \mathrm{ab}$ & 1,80 & $\mathrm{a}$ \\
16 & $8,87 \mathrm{~d}$ & $4,67 \mathrm{~b}$ & $2,51 \mathrm{bc}$ & 1,80 & $\mathrm{a}$ \\
19 & $8,59 \mathrm{~cd}$ & $3,30 \mathrm{a}$ & $2,60 \mathrm{bc}$ & $2,00 \mathrm{a}$ \\
\hline
\end{tabular}

Leyenda: Promedios con letras iguales en cada columna no son estadísticamente diferentes (Duncan, P 0.05).

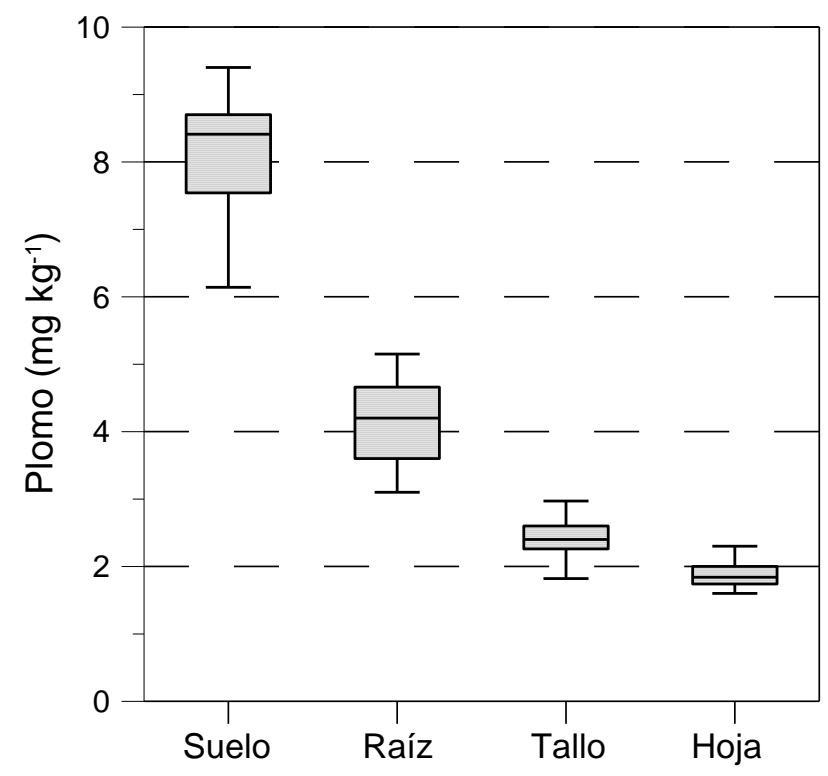

Figura 5. Concentración de $\mathrm{Pb}$ en el suelo, raíces, tallo y hojas de arroz con cáscara, medido en 5 localidades (3 repeticiones por estación) del área de estudio. 
Por otra parte en cuanto al contenido de plomo en las raíces de las plantas de arroz, analizadas, en las localidades $19\left(3,30 \mathrm{mg} \mathrm{kg}^{-1}\right)$ y $3\left(3,76 \mathrm{mg} \mathrm{kg}^{-1}\right)$ se observaron los promedios más bajos; mientras que, en las otras tres localidades (4, 5 y 16) se presentaron promedios más altos y superiores a $4,4 \mathrm{mg}$ $\mathrm{kg}^{-1}$. Al analizar el contenido en los tallos, se observa que en las localidades 3 y 5 se muestran los promedios más bajos con 2,01 y 2,31 $\mathrm{mg} \mathrm{kg}^{-1}$, respectivamente. En la localidad 4 se observó el promedio más alto $\left(2,69 \mathrm{mg} \mathrm{kg}^{-1}\right)$ y comparte el rango de significancia con los promedios valorados en las localidades $19\left(2,60 \mathrm{mg} \mathrm{kg}^{-1}\right)$ y $16\left(2,51 \mathrm{mg} \mathrm{kg}^{-1}\right)$. En relación al contenido en las hojas de las plantas de arroz, los promedios variaron de 1,80 a $2,00 \mathrm{mg} \mathrm{kg}^{-1}$ siendo iguales estadísticamente en todas las localidades. En estudios realizados por Adriano (1986) ubica al plomo y al cadmio en el grupo de aquellos elementos que presentan mayor acumulación en la parte radicular de las plantas y en pequeñas cantidades en la parte aérea. Los valores tóxicos para las hojas de las plantas están sobre los valores de de $20 \mathrm{mg} \mathrm{kg}^{-1}$, conforme reportan Wallace y Wallace (1994).

En las determinaciones de plomo del presente estudio se encontró para suelo un promedio de 8,06 $\mathrm{mg} \mathrm{kg}^{-1}$ con un valor mínimo y máximo de 6,55 y $8,87 \mathrm{mg} \mathrm{kg}^{-1}$ respectivamente, en la raíz se obtuvo un valor mínimo de 3,30 y máximo de 4,67 mg kg-1 estableciendo un valor promedio de 4,16 $\mathrm{mg} \mathrm{kg}^{-1}$, referente al tallo se determinó un promedio de $2,42 \mathrm{mg} \mathrm{kg}^{-1}$ con un valor mínimo de 2,01 y un máximo de 2,69 $\mathrm{mg} \mathrm{kg}^{-1}$ y en el caso de la hoja se encontró un valor promedio de $1,87 \mathrm{mg} \mathrm{kg}^{-1}$ con un valor minino de 1,80 y un máximo de $2,0 \mathrm{mg} \mathrm{kg}^{-1}$.

\section{CONCLUSIONES}

En base al presente estudio se concluye que las localidades estudiadas presentaron variación entre ellas debido principalmente a los contenidos de limo, arcilla, del Cd. Los análisis multivariados de componentes principales y de conglomerados mostraron congruencia en la agrupación de las localidades tanto al analizar las 12 variables estudiadas, así como al utilizar solo los metales pesados: $\mathrm{Cd}, \mathrm{Pb}, \mathrm{Cu}, \mathrm{Fe}, \mathrm{Mg}$ y $\mathrm{Zn}$. Se evidenció la presencia de metales pesados en los suelos en niveles que van de acuerdo al siguiente orden: $\mathrm{Fe}>\mathrm{Mn}>\mathrm{Cu}>\mathrm{Zn}>\mathrm{Pb}>\mathrm{Cd}$, el Hg no se lo detecto. En cuanto al $\mathrm{Pb}$ en suelos, se presentó en promedios desde 6,55 hasta $8,87 \mathrm{mg} \mathrm{kg}^{-1}$. Los contenidos de $\mathrm{Pb}$ en las raíces de plantas de arroz se presentaron en valores bajos desde 3,30 hasta de $4,67 \mathrm{mg} \mathrm{kg}^{-1}$ y en los tallos los contenidos variaron desde 2,01 a 2,69 $\mathrm{mg} \mathrm{kg}^{-1}$. En relación al contenido en las hojas de las plantas de arroz, los promedios variaron de 1,80 a $2,00 \mathrm{mg} \mathrm{kg}^{-1}$ siendo iguales estadísticamente en todas las localidades. Se evidenció la presencia de plomo en los diferentes órganos de la planta de arroz encontrándose en raíz, tallo y hojas, niveles no tóxicos para el cultivo.

\section{AGRADECIMIENTOS}

Dejamos constancia de nuestro agradecimiento al Dr. Jan Feyen por su crítica positiva en la conclusión de este artículo, también al Dr. Eison Silva por sus cometarios en aplicaciones estadísticas.

\section{BIBLIOGRAFÍA}

Adriano, D.C., 2001. Trace elements in terrestrial environments: Biogeochemistry, Bioavailability, and Risks of Metals, Springer, New York, 2001. En: Baird, C., 1999. Descargado de servlet http://www.abebooks.com/SearchResults? an=Adriano\%2C+D.C. \%3BAdriano\%2C+Domy+C.\% 3 BAdriano $\% 2 C+$ The + D. + C. + Trace + Elements + in + Terrestrial + Environment el 2 de mayo 2010.

Adriano, D., 1986. Trace elements in terrestrial environments. New York, USA, 533 págs. 
Allison, L.E., H.E. Hayward, L.A. Richards, L. Berntein, M. Fireman G.A. Person, L.V. Wilcox, C.A. Bower, J.T. Hatcher, R.C. Reeve, 1973. Diagnóstico y rehabilitación de suelos salinos y sódicos Trad. L.A. Richardas, 6a Ed., LIMUSA, México, 172 págs.

Arcos, V., R, Castro, 2005. Metales pesados en aguas sedimentos y organismos. Rev. Científica de Ciencias Ambientales, 101, 103-120.

Baird, C., 1999. Environmental chemistry. 2nd ed. Freeman. New York, NY, USA. Descargado de http://www.amazon.com/Environmental-Chemistry-Colin-Baird/dp/1429201460 el 5 enero 2011.

Bernal, M.P., S.C. Vazquez, D.J. Walker, 2007. Aplicación de la fitorremediación a los suelos contaminados por metales pesados. Aznalcóllar Ecosistemas, 16(2), 67-76.

Bonnet, J., 1960. Edafología de los suelos salinos y sódicos. Estación Experimental Agrícola, Universidad de Puerto Rico, 283 págs.

Brookes, P.C., 1995. The use of microbial parameters in monitoring soil pollution by heavy metals. Biol. Fert. Soils, 19, 269-279.

Brunetti, P., G. Soler-Rovira, K. Farrag, N. Senesi, 2009. Tolerance and accumulation of heavy metals by wild plat species grown in contaminated soils in Apulia region, Southern Italy. Plant Soil, 318, 285-298.

Cataldo, D.A., R.E. Wilding, T.R. Garland, 1987. Speciation of trace inorganic contaminants in plants and bioavailability to animals: an overwiew. J. Environ. Qual., 16(4).

C.I.M.M. Tecnologías y Servicios, 2010. Estudio de contaminación de cenizas y suelos en la ciudad de Chaitén y propuesta de medidas de mitigación y restauración. Descargado el 24 de septiembre 2010 de http://seremiloslagos.redsalud.gov.cl/url/item/9abf7df1a3c422e5e04001011f0104a2.pdf.

EPA (United States Environmental Protection Agency), 1994. Method 3051 Microwave assisted acid digestión of sediments, sludges, soils and oils. Revision 0. In: Test methods. SW-846. Section A, Part I, Chapter 3, 3051, 1-14.

Estévez, J., M.L. Andrade, P. Marcet, M.J. Montero, 2000. Fijacion y movilidad de Cadmio y Zinc en tres tipos de suelos ácidos de Galicia, España. Ciencias del Suelo, 18(1), 28-35.

Gulson, B.L., K.J. Mizon, M.J. Korsch, D. Howarth, 1996. Non-orebody sources are significant contributors to blood lead of some children with low to moderate lead exposure in a mayor mining community. The Sci. Total Environ., 181, 223-230.

Hernandez, F., J. Medina, J. Ansuátegui, 1990. Contaminación por metales pesados en el Mediterraneo: Aplicación a Organismos Marinos del Litoral de Castellón. Diputació de Castellón, 160, 21-38.

Henríquez,. C., G.A. Cabalceta, 1999. Guía practica para el estudio introductorio de los suelos con un enfoque agrícola. ACCS, San José, Costa Rica, 111 págs.

Jackson, M.L., 1970. Análisis químico de suelos y plantas. Trad. del Inglés por José Beltrán Martínez 2da Ed. OMEGA, Barcelona, 662 págs.

John, D.A., J.S. Leventhal, 1995. Bioavailability of metals. Descargado de http://www.unalmed.edu.co/rrodriguez/MODELOS/depositos-ambiente/BioaviabilityOf Metal.pdf/ el 17 de abril 2011.

Kabata-Pendias, A., H. Pendias, 2004. Soil-plant transfer of trace elements: An environmental issue. Descargado de http://www.sciencedirect.com/science?_ob=ArticleURL\&_udi el 27 de septiembre 2010.

Kashem, A.M., B.R. Singh, 2001. Metal availability in contaminated soils: I. Effects of flooding and organic matter on changes in $\mathrm{Eh}, \mathrm{pH}$ and solubility of $\mathrm{Cd}, \mathrm{Ni}$ and $\mathrm{Zn}$. Nutr. Cycl. Agroecosys., $61,247-255$.

Liu, J.G., K.Q. Li, J.K. Xu, Z.J. Zhang, T.B. Ma, X.L. Lu, J.H. Yang, Q.S. Zhu, 2003. Lead toxicity, uptake, and translocation in different rice cultivars. Plant Sci., 165, 793-802.

Majer, B.J., D. Tscherko, A. Paschke, 2002. Effects of heavy metal contamination of soils on micronucleus induction in Tradescantia and on microbial enzyme activities: A comparative investigation. Mutation Res., 515, 111-124. 
Montenegro, G., C. Fredes, E. Mejia, C. Bonomelli, L. Olivares, 2009. Contenido de metales pesados en suelos cercanos a un relave cuprífero chileno.Descargado de http://scielo.org.mx/pdf/agro/ 134n4a9.pdf el 10 de enero 2011.

Navarro-Aviño, J., P.A.S. Alonso, J.R. López-Moya, 2007. Aspectos bioquímicos y genéticos de la tolerancia y acumulación de metales pesados en plantas. Ecosistemas, 16(2), 10-25.

Oficial Methods of Analysis of AOAC INTERNATIONAL (OMA), 18th Edition, 2005. AOAC Official Method 999.10. Descargado de http://sutlib2.sut.ac.th/sut_contents/H125800.pdf el 26 de septiembre 2010.

Pérez, C.I., A.M. Moreno, J.P. González, 1995. Influencia de la fracción arcilla y arena en el contenido y disponibilidad de metales pesados en el suelo. Edafología: Sociedad Española de las Ciencias del Suelo, 1, 83-89.

Pozo, W., 2001. Programa de educación e investigación en agricultura tropical sostenible en el Ecuador. Fondo Competitivo de Investigación Agropecuaria 2002. Portafolio de Alianzas Estratégicas Internacionales y Proyectos de Investigación PROMSA, 120 págs.

Pozo, W., T. Sanfeliu, G. Carrera, 2010. Variabilidad espacial temporal de la salinidad del suelo en los humedales de arroz en la Cuenca Baja del Guayas, Sudamérica. RTE, 23(1), 73-79.

Rodríguez, R., 2010. Situación arrocera Ecuatoriana. Corporación de Industriales arroceros del Ecuador. CORPCOM, 25 págs.

Ross, S.M. 1994 Toxic metals in soil-plant systems. Descargado de http://www.cabdirect. org/abstracts/19941904711.html;jsessionid=042B79D9FA54CCCD3B385006 A73DD625 el 12 de enero 2011.

SICA, 2001 Servicio de información y Censo Agropecuario, 2001. Sistemas y Procedimientos de Gestión. Interna Ambiental. Identificación de mercados y tecnología para productos agrícolas no tradicionales de exportación, ambientales para la producción agrícola. Quito, Ecuador. Descargado de http//:www.sica.gov.ec/agronegocios/Biblioteca/Convenio\%20MAG\%20IICA/ consid_mag.pdf el 27 de septiembre 2010.

Torres, G., E.A. Navarro, J. Languasco, K. Campos, N. Cuizano, 2007 Estudio preliminar de la fitoremediación de cobre divalente mediante Pistia stratioides (lechuga de agua). Descargado de http://cienciaescolar.net/proyectos/wpcontent/uploads/2009/09/

Art_14_Torres_lechuga_agua.pdf el 15 de diciembre 2010.

UEFC (Unidad Ejecutoria del Fondo Competitivo), 2002. El Ecuador Consolida su Fondo Competitivo de Investigación Agropecuaria 2002. Portafolio de Alianzas Estratégicas Internacionales y Proyectos de Investigación PROMSA adjudicados desde 1999 al 2001, Quito, Ecuador. UEFC, 56 págs.

Walkey, A., I.A. Black, 1934. An Examination of Degtjareff method for determining soil organic matter and a proposed modification of the chromic acid titration method. Soil Sci., 37:29-37.

Wallace, G., A. Wallace, 1994. Lead and other potentially toxic heave metals in soil. Communication in Soil Science and Plant Analysis, 25, 137-141.

Wilcke, W., H. Döler, 1995. Schwermetalle in der Landwirtschaft. Kuratorium fur Technik und Bauwesen in der Landwirtschaft, Darmstadt, Germany, 98 págs.

Yao, H.Y., Z.L. He, M.J. Wilson, C.D. Campbell, 2000. Microbial biomass and community structure in a sequence of soils with increasing fertility and changing land use. Microbial. Ecol., 40, 223237. 